Please do not remove this page

RMIT

UNIVERSITY

\title{
Getting a GRiP: examining the outcomes of a pilot program to support graduate research students in writing for publication
}

Cuthbert, Denise; Spark, Ceridwen

https://researchrepository.rmit.edu.au/esploro/outputs/9921858573201341/filesAndLinks?institution=61RMIT_INST\&index=null

Cuthbert, D., \& Spark, C. (2008). Getting a GRiP: examining the outcomes of a pilot program to support graduate research students in writing for publication. Studies in Higher Education, 33(1), 77-88. https://doi.org/10.1080/03075070701794841

Document Version: Accepted Manuscript

Published Version: https://doi.org/10.1080/03075070701794841

Repository homepage: https://researchrepository.rmit.edu.au

(C) 2008 Society for Research into Higher Education

Downloaded On 2023/04/26 11:43:27 +1000 
Thank you for downloading this document from the RMIT Research Repository.

The RMIT Research Repository is an open access database showcasing the research outputs of RMIT University researchers.

RMIT Research Repository: http://researchbank.rmit.edu.au/

\section{Citation:}

Cuthbert, D and Spark, C 2008, 'Getting a GRiP: examining the outcomes of a pilot program to support graduate research students in writing for publication', Studies in Higher Education, vol. 33, no. 1, pp. 77-88.

See this record in the RMIT Research Repository at:

http://researchbank.rmit.edu.au/view/rmit:15647

Version: Accepted Manuscript

Copyright Statement: (c) 2008 Society for Research into Higher Education

Link to Published Version:

http://dx.doi.org/10.1080/03075070701794841 


\section{Getting a GRiP: Examining the outcomes of a pilot program to support Graduate Research Students in Writing for Publication}

Language is learned by participating in human relationships ... the language of expertise is learned by performing that expertise. (Leverenz, 2001, 59).

\section{Introduction}

In the increasingly competitive context of the modern university, writing groups have emerged as one way of developing the research potential of academic staff. Research in this field to date (see Galligan, Cretchley, George, McDonald, McDonald and Rankin, 2003, 28) points toward the important role writing groups play in fostering academic writing and research development. In their study of writing groups, for example, David Boud and Alison Lee (1999) critique the assumption that writing is the 'end' of the research process and argue that it would be more useful to conceive of the processes of review and analysis as part of the day to day localised practice of academic work. They further suggest that writing groups 'clearly worked to reposition participants as active scholarly writers within a peer-learning framework’ $(1999,9)$. Boud and Lee $(2003,190)$ have also examined the role writing groups play in identity formation, particularly their capacity to 'help [academics] locate themselves in a productive relationship to change'. Such change includes the increased emphasis on research productivity across the higher-education sector.

Other articles in the research literature on writing groups in the Australian and international contexts support the finding that writing groups enhance academics’ research and publication skills. In their study of writing groups from three universities, Galligan et al (2003) show that the outcomes of staff participation in 
these were increased publication output, the collective discussion of writing processes, increased understanding of collaborative work processes and overall increase in levels of work satisfaction. While acknowledging that any writing group will develop its own themes, strengths and weaknesses and that some will fail at certain stages or under the influence of particular convenors, these authors highlight the overall strengths of the group of which they were a part, including: a stimulating mix of disciplines; goal-focused activities; a group that was nurturing rather than demanding or threatening and good timing for participants involved in or about to embark on academic writing (Galligan et al, 2003, 38). These generally positive findings are supported by Morss and Murray’s $(2001,35)$ study which claims that 'a structured programme in writing development can enable academics to improve their written output and writing process.' These authors also highlight the value of writing for publication programmes in the context of the absence of research on, and support for, academics aiming to improve their publishing profiles.

As the above discussion shows, there is a small but growing body of research on writing groups for academic staff. However, there is almost nothing on writing groups for graduate research students. This may be because there are very few writing for publication programs designed and run specifically for graduate research students; or, where they are run, these have not been documented. Even universities with dedicated writing centres, report that graduates receive 'little assistance' to learn new ways of writing (Garbus, 2005, 172). Though academic managers have begun to see 'the need for the development of staff in the research role in the light of growth of higher education and changes to the sector in many countries' (Boud and Lee, 2003, 187), 
the evidence suggests that, overall, this vision has not yet been extended to include graduate students, or has remained undocumented.

As with the provision of programs, the issue of writing for publication by graduate research students in the humanities and social sciences is almost untouched in higher education, discipline-based and academic-writing research literatures. Our research goes some way in filling this gap. This article presents findings from research undertaken with participants from humanities and social science disciplines in a Faculty-based writing for publication pilot project called GRiP (Graduate Researchers in Print). In this paper, we draw on academic literature on academic writing groups, evaluations undertaken with GRiP participants, and our own experiences and perceptions from, respectively, the perspectives of an Associate Dean (Graduate Research) charged with the overall management of higher degree by research education and the convenor of GRiP, with responsibility for the delivery and evaluation of this program. The program and research were conducted in 2005. The findings indicate that the provision of a structured program to support research candidates develop writing for publication is effective in terms of assisting candidates to publish their research; and a useful adjunct to discipline-specific research supervision and training provided in School and Departmental settings.

In the following we describe the GRiP program, provide some discussion of the context in which it was established and outline the method employed in our research. As our research method is qualitative, the data provides rich insights into the experience of graduate research students, their perceptions of the research process and their role as 'researchers' and 'writers'. The main body of the article considers the 
major finding of our research with the participants in the GRiP program, demonstrating that GRiP played a significant role in developing graduate students' sense of themselves as researchers with the capacity to contribute to the scholarly community. We then consider the implications of these findings for the provision of graduate research education in the humanities and social sciences. Before embarking on a discussion of the GRiP program, however, it is necessary to outline the context in which the program developed and operated.

\section{Graduate Research Education in the Australian context}

The reasons for the lack of graduate publication programs are not clear and, in the absence of literature on the subject, we resort to informed speculation. That there appears to have been no widespread move (at least within the humanities and social sciences), to develop the publishing potential of graduate research students may be due, in part, to a lack of clarity in universities about what the outcomes of graduate research education should be (or a fond belief that these outcomes are self-evident and thus not in need of articulation) (Sheely, 1996). Another factor which seems to be at work, at least in humanities disciplines, is that the relatively individualistic writing and publication culture which prevails may not be as conducive to discussions about the processes of writing for publication as the cultures in disciplines in which joint publications, including co-authoring arrangements between supervisor and candidate, occur more frequently. This publication culture is the norm in most scientific and technical disciplines, such as Science, Engineering and Medicine. It is also probably the case that the issue of graduate publication in Australian higher education has been somewhat at the mercy of different policy regimes and their funding formulae. 
Overwhelmingly, the imperative which has shaped the policy and management of higher degree by research education in Australian universities in the period since 2001 has been the funding formula of the Research Training Scheme (RTS) which places unprecedented pressure on two areas in the management of graduate research education: attrition and timely completion. The RTS, coupled with the Institutional Grants Scheme (IGS), has worked to downplay the importance of publications in the research funding awarded to universities relative to the importance of higher degree by research completions and load (total research degree enrolments).

In this way, the RTS has prompted more active management of higher degree by research candidates than previously. This management has been directed to working improvements in those areas to which the RTS funding formula is particularly geared: the stemming of attrition and the attainment of timely completions. Arguably, this has occurred at the expense of other areas in the delivery of graduate research education, such as actively inducting candidates into the publication cultures of their disciplines. In this regard, the timing of the RTS appears to have cut across other developments in the field, including the clamours of students themselves for greater levels of professionalisation to assist their post-graduation career development (Guillory, 1996, 4).

Simultaneously, other developments in Australian higher education, such as the widespread introduction of $\mathrm{PhD}$ by Publication programs across the sector in the period since the late 1990s (see Sheely, 1996) appear to be placing additional emphasis on the research and publication potential of graduate research students. The hitherto undervalued potential of graduate research students in the humanities and 
social sciences as writers and publishers is thus likely to be increasingly addressed as universities recognise this largely untapped capacity to enhance an institution’s research profile and publication output; and through the PhD by Publication route secure both degree completions and increased publications simultaneously.

That the provision of a structure in which graduate research students may learn the tools of the academic trade - including publishing skills - has not been seriously addressed seems especially noteworthy when we consider the findings from the one article on graduate writing groups that we could locate; namely that seven of the eight member writing group wrote, submitted or published a paper during their membership compared to only two of ten not in a writing group (Page-Adams, Cheng, Gogineni and Shen, 1995, 405). In a call for institutional support of graduate writing groups, Page-Adams et al also cite research showing that 'new faculty members who learned to balance writing, teaching and collegiality early in their academic careers had relatively high levels of publication productivity’ (Page-Adams et al 1995, 406). Research on graduate experience in writing centres also suggests the benefits of 'peer' and ‘collaborative' learning. Following Brufee (1984), Carrie Shiveley Leverenz (2001, 54) argues that 'by working in peer groups, with the guidance of a teacher, students could learn to write in ways associated with academic expertise'. Leverenz (2001, 58) also notes that: ‘[w]riting centers provide an opportunity for graduate students to build relationships with other writers, relationships based on conversations about ideas and about writing that might be seen as rehearsals of expertise'. In light of 'vested interest [among some academics] in limiting that expertise' (Leverenz, 2001, 56), it seems possible that the limited support hitherto granted to graduate students when it comes to writing and publishing may reflect an 'intentionally exclusionary' 
(Leverenz, 2001, 56) culture in which graduate publishing is perceived as constituting a threat to the 'expert' status of faculty members. As David Damrosch argues in a related context, whatever else it may be about, graduate research education is also frequently about power, privilege and policing the gateways to these (Damrosh, 2006, p.38).

While not dealing with student writing groups per se, John Guillory introduces a salutary note to the subject of graduate research candidates as writers for publication with his concerns about the growing phenomenon in some US graduate schools which he dubs ‘pre-professionalism’ (1996). For Guillory, the new 'professional domain’ of the 'pre-professional' has been brought about through 'the penetration of graduate education by professional practices formerly confined to later phases of the career, the most obvious examples being publication and the delivery of conference papers' (Guillory, 1996, 4). Guillory (1996, 4-8) reports the concerns of senior academics in the field of literary studies, concerning the degree to which such premature or 'precocious' professionalism in research candidates has resulted in a 'deformation' of graduate education, by, for example, inhibiting students from embarking on 'longterm intellectual projects' as they keep one eye on opportunities to publish. Guillory's concerns should be balanced, however, against findings which indicate that the capacity or support to publish during candidature is reported on positively by research candidates evaluating their research degree experience (Heath, 2002).

\section{The University Context of the GRiP Program}

The GRiP (Graduate Researchers in Print) program was initiated and established in 2005 by Denise Cuthbert in her role as Associate Dean (Graduate Research), with the 
aim of supporting higher degree research candidates in the Arts Faculty to commence and develop scholarly publications. The primary aims behind the scheme were student-centred, to: enrich the candidature experience; enhance the sense of professionalisation amongst candidates; add to their CVs and increase their competitiveness in the academic labour market post-graduation. Notwithstanding concerns that time spent on publications was time taken away from the completion of this thesis, it was hoped that success in publishing would work to enhance the commitment of candidates to their research projects and confidence in themselves as researchers and, in this way, assist more candidates to successfully bringing their research degrees to completion.

It must also be acknowledged that in the present funding environment in which institutions earn research income, from publications in approved categories, albeit at a now reduced rate under the RTS, the process of securing Faculty funding for the pilot project was not solely student-focused. A one-page document was prepared by the Associate Dean which lined up the proposed cost of mounting the pilot scheme with the financial returns to Faculty if $\mathrm{x}, \mathrm{y}$ and $\mathrm{z}$ number of participants produced publications within one year of their participation. The numbers were persuasive and funding was secured. In addition, the rate at which research candidates in the Faculty published, either as sole authors or conjointly with their supervisors, did not compare favourably with rates in other Faculties. For instance, 2004 Research Quantum (the government scheme which audits research publication and distributes funding according to the numbers of publications in approved categories) data showed that only seven percent of the Arts higher degree by research cohort had published in 
approved categories in that period, compared with over 25\% in some science and technology faculties.

\section{GRiP: Description of the Program}

The GRiP program in 2005 consisted of 27 participants divided into four groups each comprising 6-9 people which met once a month for two hours in a meeting room within the Faculty. With the exception of two participants who were very recently completed graduates with no prior history of publication, all were currently completing a research Masters or PhD in Arts.

GRiP participants came from various disciplines in the Faculty of Arts, a large and diverse Faculty comprising humanities, social and environmental sciences, languages and performing arts. The decision to run multi-, rather than single-, discipline groups was based partly on the diverse backgrounds of participants. In addition, it was influenced by the expectations, informed by the research literature (Galligan et al, 2003), that one of the advantages of multi-disciplinary groups is additional encouragement for participants to focus primarily on the quality of the writing, rather than on the specialised aspects of the content. The facilitator, Ceridwen Spark, also felt that the discipline-based academic supervisors would (or should) be filling the role of 'expert' content readers.

The groups began in March 2005 and ran until December of that year, with each group meeting a total of nine times. The purpose of GRiP was to encourage students to write and publish and, to this end, the program employed peer-support and review in a workshop setting. Students were required to draft an article or chapter to be read 
and discussed by the group and to read the drafts of other members of their group. The time commitment for GRiP participants was attendance at one two-hour workshop per month and the time required to draft material and read the work of other group members.

At the initial meeting, a timetable was drawn up for the presentation of group members' work. Each participant was required to commit to a time to present a piece of writing to the group. Considerable time, in the first session and in subsequent meetings, was spent setting up and re-inforcing the processes and the expectations of both the drafting and peer-review process. The facilitator discussed the method by which the material would be distributed and how group discussion would proceed, emphasising the need for constructive criticism. An essay on the history and function of writing groups was distributed for interested students to read in their own time (see Gere, 1987, Chapter 3). For the second session, students were asked to prepare an abstract describing the article they would write over the ensuing months and to identify 1-2 journals in which they aimed to publish. This enabled GRiP participants to develop a writing goal as well as giving them time to familiarise themselves with one another.

For the sessions in which students’ writing underwent peer-review, the following protocol was followed. Each writing draft presented to the group was to be accompanied by a short list of questions and guidelines for reading so that other group members would know what specific struggles or concerns the author had about the paper. Papers were to be distributed via the facilitator a week before the monthly meetings. At the meeting itself, students were asked to come having read the draft and 
with written comments on issues including structure, clarity, flow, direction, worthiness and readability; in addition, they were asked to respond to the particular issues about the paper raised by the author. At the meeting, the author introduced the work briefly, for example, talking about its history and the journal being targeted for its publication. The author was then asked to listen without interrupting as group members conducted an initial discussion of the article, the aim being to allow the author an opportunity to ‘eavesdrop’ on a conversation about their work without being tempted to defend and explain it. After this initial 'eavesdropping' period, the author joined the discussion and had the opportunity to ask and answer questions about the article.

Over the nine months, the facilitator also ran sessions on aspects of publishing, including identifying appropriate journals, preparing submissions, responding to reviewers' reports, writing book proposals, turning theses into books and writing for the non-academic media. In these ways, GRiP took an inclusive approach to improving graduate writing while still emphasising the production of peer-reviewed journal articles and book chapters. This inclusiveness reflects that the primary aim of GRiP was to develop graduate writing skills, with the emphasis being on understanding that writing must always have in view a particular audience. Within this overall aim, the production of scholarly publications for the enhancement of the research profiles and CVs of participants remained a key objective of the program. A secondary aim was enhancing faculty overall publication outcomes.

\section{Research Methods}


The data for this study were collected in 2005 by means of four 20-40 minute focus group discussions conducted by the author and group facilitator, Ceridwen Spark, at the final session of each GRiP group meeting. Focus groups are fundamentally about group interaction and over the course of the year individual GRiP participants inevitably participated in a range of exchanges, including critique and disagreement with one another and the facilitator. The focus group method was therefore chosen as the ideal way of gaining feedback because it was a natural and logical extension of the dynamic that already existed between the group members and the facilitator. Thus while it is theoretically possible that the facilitator, by acting as researcher, skewed responses, we consider this risk was minimised by the open, reflectively critical mode of communication that was by then well-established between herself and group members. All participants were volunteers; and the research was approved by the university’s Standing Committee on Ethical Research.

There were four focus groups each made up of 5-6 GRiP participants who had consented to participate in the research, a total of 20. In order to minimise the risk of coercion, an independent Faculty employee did the initial call for participants. This entailed providing the students with an explanatory statement detailing that their participation in GRiP in no way required them to take part in the research. Only those students who consented to participate were subsequently approached to do so. During the focus groups, participants responded to questions around the themes of writing for publication and the extent to which GRiP had facilitated this process; this included questions about publishing, such as how they felt about it prior to, and after, participating in the GRiP program. Participants were asked to discuss what they found most helpful and most difficult about their involvement in the program, 
including any difficulties they had encountered with the peer-review process. These group discussions were recorded and later transcribed for the purposes of analysis. The researchers then identified key themes that emerged across all groups. In the following analysis, all participants have been assigned pseudonyms by the authors to ensure anonymity.

\section{GRiP Outcomes}

Overall, GRiP participants were positive about their involvement in the program. For instance, no group member expressed anything negative about the experience of peer review. Given the intimate link between writers and their work - such that criticism of one’s writing may be felt as 'an attack on the self' (Gere, 1987, 103) - this is a notable finding.

The program also had positive 'hard' outcomes (Morss and Murray, 2001, 35) as revealed by a survey at the end of 2005. For instance:

- $98 \%$ of 2005 GRiP participants drafted an article for eventual publication in a referred journal;

- $53 \%$ submitted an article for publication;

- $19 \%$ had journal articles accepted (all others were waiting on reviewers' reports);

- $19 \%$ had started a draft of a chapter for publication in an edited book collection and

- 23 \% had conference papers accepted for publication in an edited conference proceedings or collection. 
In addition, GRiP participants reported other benefits or 'soft outcomes' (Morss and Murray, 2001, 35.) including: a sense of being 'supported' instead of 'pressured' to publish; confidence that they knew how to get published; a sense of community with other postgraduates and increased awareness about what makes a good article. In the next section these findings are discussed in greater detail with participants' evaluations summarised in relation to three key themes emerging from the data; demystification of the writing process, writing for an audience and support versus pressure to publish.

\section{Secret Magical Stuff: Demystifying publication}

A key finding of the study is that GRiP demystified the publishing process for graduates. In this section, we discuss the anxiety expressed by graduate students and their perceptions of the culture of 'secrecy' surrounding the processes of getting published which the program helped to dispel. Subsequently, we consider how elucidating the writing process contributed to graduates' emerging sense of confidence that publishing was an achievable goal.

When asked to reflect on what they felt about publishing prior to participating in GRiP, participants used words like 'mystery', ‘secrecy’ and 'privacy’. In their preGRiP experiences and perceptions, writing and thinking about publishing were, in effect, things which took place in isolation and not readily shared with others. For instance, Emma comments:

I found it probably a bit more mysterious than I see now, the process of publishing in academic journals, so it was more something I'd just done privately ... I hadn't talked to others about it. 
Glenn and Karen support this notion of isolation saying respectively: 'there's a sense in which ... the only person who ever read your work is you ... and it's effectively a private activity' and 'before this year ... I was just so personal about my writing'. This privacy or isolation is closely related to graduates' ignorance about how to get published. For example, recalling an ostensibly supportive information session on publishing in which the audience of graduate students was told to 'publish, publish, publish!' Nick reports that he felt 'well I just don’t know how'. This was confirmed by Sarah who commented that she felt 'overwhelmed and intimidated' and Siobhan who remarked 'I suppose I was ... quite ignorant of the whole process, probably quite daunted'. In this context of ignorance about the process of publication, some of the fear graduates feel heightened anxiety about the process of peer review. Glenn's discussion of this connotes the mystery that graduates associate with the publishing process. He says that working toward publishing involves: 'exposing yourself to the potential for criticism ... because you're sending your work off to these mysterious elders of the academic community who are going to criticise you.'

In our view, GRiP participants' sense of being 'ignorant' and 'daunted' is inseparable from, and causally linked, to the general silence surrounding publication as process within the academy. As Tessa puts it publishing is 'of one of those things that people don't talk about ... you're just supposed to know how to write papers'. Indeed, the only thing students report hearing loud and clear is the injunction to 'publish, publish, publish', as Nick summarised it. This silence appears to have much to do with a culturally dominant view of the author as a privileged individual intellect working in isolation (see Gere, 1987), a notion that apparently underpins the unwillingness 
among some academic staff to talk with graduates about publishing in a way which demystifies the processes and allows students to see these as a normative and achievable part of the research process. Kim’s comments support the idea that academics are reluctant to discuss publishing. She notes: 'everyone gets bad reviews [i.e. reader's reports], they just don't like to talk about it!' Shrouded in silence and even shame, negative experiences such as the 'bad reviews' Kim mentions contribute to graduates’ perception of publishing as something to be feared and about which to remain silent.

One of the most important functions of the GRiP program, then, was to lift the shroud from, and challenge the silence and anxiety surrounding, publication. If the silence and mystery surrounding publication are inextricably related to individualistic notions of the author as an 'inspired being [carrying] out the dictates of the muse' (Gere, 1987, 58), challenging this notion involves bringing the social and practical dimensions of writing for publication to light. Being a social and dialogic activity, the process of peer review that characterised GRiP counteracted the myth of the isolated author for whom the publishable work 'arrives' ready-made. In short, the process exposed both the hard work of writing; and its sociality. That is, that writing is always a collaboration between writers and readers and not simply the isolated activity of an individual.

GRiP participants highlighted the relationship between learning about other group members' struggles with writing and their own developing confidence. The following comments, which are characteristic of the focus group discussions on this theme, illustrate this: 
For me the benefit of GRiP was seeing other people struggling with the practice of writing and working through those issues. I found it most useful because I realised, I wasn't so far behind where other people were. Other people were struggling working through stuff and I could do that too. (Nick)

Seeing people work through stuff ... willingly struggle with stuff. Like I ...had in my mind that you don't start writing until you're ready; 'til you know what you want to say ... once you do start writing you realise you don't know what you want to say it's going to take a lot of time and work to do that. Watching other people do that gave me the confidence to think, ok I can do that. We all have to go through this, it's ok. (Glenn)

By observing and helping other group members to develop their writing, GRiP participants learned that getting published, involves a number of practical steps rather than 'magical ingredients' (Elizabeth).

Over the year and particularly in specific sessions, for example, the session on submitting articles and responding to reviewer's reports, GRiP participants learned more about the steps involved in writing an article. Consequently, they began to conceive of publishing less as a daunting impossibility than as something they could do if they followed these steps. Discussing the session on submitting articles and responding to reviewer’s reports, for instance, Emma noted:

And I did find it very valuable when you (i.e. the facilitator) talked about, showed letters you'd got from journals and went through and explained that some of the responses that seemed like a negative response in fact it was positive, and you know, the kind of difference between what your assessment was of the quality of the work and sometimes how a journal responded. So that to me was really interesting ... I think often that stuff's quite secret. That academics would keep to themselves, and academics who review other people’s work 
wouldn't talk about those things. So that's been really helpful. So that has changed it for me, just to see it's another process - there's a series of steps that you have to go through and success isn't guaranteed, but it is possible. So that's been the change, I guess, for me.

Emma's identification of the reviewing process as 'secret' accords with the other statements GRiP participants made about the way publishing is represented (or not) within academia. GRiP enabled Emma to see inside this 'secret' process and identify the 'series of steps' involved in publishing, thereby demystifying it and making it 'possible'. Elizabeth spoke about learning that 'publication isn't something that sort of happens from up here, it's not a magical thing that, you know, the expert has all the knowledge and they just rip out a paper which is ready - it's actually something that's... it's a grounded thing, you start with something and you have to work it up'. Nick's statement pithily sums up this transformation in thinking about publishing; 'I feel clearer now on what that process is. It's not that you suddenly wake up in the morning and you've written an article but that's kind of ... how I thought it worked before’.

No longer conceived of as a magical process that is seldom spoken of, publishing has become a ‘less mysterious [process involving] steps to go through’ (Deb). Thus, for GRiP participants, increased knowledge about the practical steps involved in publishing is related to growing confidence. In the next section, we focus our discussion on what GRiP participation taught graduates about writing for an audience.

\section{Writing for an audience}

The second key theme to emerge from the data was the importance of writing for an audience. GRiP participants spoke about the ways the program assisted them to think 
about writing for other people. Indeed Siobhan noted this to be the most important thing she gained from participating in GRiP, saying 'that's what I learnt the most, was the actual experience of writing for an audience'. For participants, subjecting their work to the process of peer review was an important and safe way to open themselves up to criticism of the kind that might emerge from potential reviewers. Glenn, for example, suggested:

You've got to try and ... expose your work more because it makes your work better but you need to do it in little baby steps because you don't want to throw yourself completely out there, it's a good way of introducing you to that process of review.

This was supported by Nick who commented that there were very few opportunities to get this feedback:

The very open structure of a research degree means that there are few times in which you are exposed to scrutiny and when you are you are expected to be at a very high level. It's nice to have more points along the way at which you can stop and rest and reconsider how you're going.

GRiP participants also commented that the process of reading and critiquing others' work enabled them to improve the ways they read and critiqued their own. Sarah, for example, noted that the process of commenting on others' work enhanced her own writing:

I learnt a lot about actually approaching a piece of work and marking it in a way. Like I actually feel like I've come out of this with a much greater, ability ... to see where I'm not making sense.

Jan also commented on the process of critiquing others' work: 
I think it was really good listening to how everyone praised and critiqued everyone in turn and then when it's your turn to sit down and think about writing, to try and draw on those experiences to make your own article better too.

This process of turning the critique one has practiced on the writing produced by others back on to one's own work reflects a transition in the writer's mind and identity. No longer individuals writing in isolation, GRiP participants quickly developed a sense of the need to connect with, and make sense to, readers. This transition is discussed by several writing group scholars, including Moffett who notes: 'the responses of writing groups foster intellectual development because they help students learn to move beyond egocentrism to take the perspectives of others' into account, (Moffett cited in Gere 1987, 23). By providing graduates with a 'real audience', GRiP thus served as an important reminder that 'language is learned by participating in human relationships’ (Leverenz, 2001, 59), something which is apt to be forgotten in the highly specialised and frequently isolated realms inhabited by academics and graduate researchers.

GRiP participants' responses beg the question: 'why is the supervisor not considered an audience'? The answer seems to inhere in the graduate students' belief that there was a need to go beyond the supervisor in order to get a sense of what a wider audience would think of the work. This makes sense in light of the ultimate need to submit one’s thesis to external supervisors. Georgina commented: ‘it’s just really good to get that feedback because you're going to have to go out beyond your supervisor.' In this regard, the GRiP peer-review process appears to have provided an important and enabling intermediary step for participants between writing for their 
supervisors and writing for an audience well beyond this relatively closed supervisorcandidate relationship.

Whether or not graduates trust their supervisors to act as a sufficiently critical audience for their writing, their participation in GRiP gave the majority of participants in 2005 a keen sense of the need to write for an audience beyond the one or two people who were already engaged in critiquing their work. The benefits of this, in terms of thinking critically about writing as a process of communication with that wider audience, were, likewise felt by the majority of participants. Moreover, because the program provided peer review in a supportive peer setting, rather than an intimidating one characterised by the inevitable power inequity between supervisors and graduates, it enabled participants to accept and respond to criticism in ways that were positive and helpful, as distinct from demoralizing and disabling. The issue of support versus pressure and the relationship between graduates and their departments and supervisors are considered in the next section which highlights the way GRiP enhanced participants’ sense of belonging to an academic community.

\section{Support versus pressure to publish}

One of the main things GRiP participants appreciated about their involvement in the program was the opportunity it gave them to develop a sense of collegiality with other graduates. Speaking about the isolation of doing a research degree Sarah said 'I feel a bit isolated within my discipline ... like it’s just me doing my research’. The participants also connected this isolation to a feeling of being pressured to publish. Kim, for example, mentioned 'this whole pressure to publish and publish and publish' in terms of 'the kind of isolation of that moment' and Bronwyn recalled how slow and 
difficult the process of trying to publish was 'when [she] was doing it all on [her] own'.

Counteracting their sense of isolation, GRiP afforded graduates the reassurance that they were all in it together. Glenn, for example, spoke about the importance of realising:

You're not the only person who's screwed here, like everybody's grappling with these concerns and you can empathise with them but you can also kind of relax because it's nothing that you would be facing on your own. I think it's a very good way of building that sense of you being a community of scholars and a community of writers, rather than an isolated person who has to fight their way in to the community; an academic or scholarly community.

The experience of publishing is often construed as a moment of arrival: endowed with the secrecy and mystery we discussed earlier. It is equated with a conferral of legitimacy by those who are already seen as 'initiated' insiders. Glenn’s description of 'an isolated person who has to fight their way in to the community' connotes the antipathy and competition that graduates associate with publishing and indeed with academia more generally - an unfortunate consequence of the increasing pressures on funding and career advancement in the sector to which our graduate students are inevitably exposed. Against this, GRiP provided a sense of togetherness and solidarity, a welcome sense of collaboration rather than competition. Highlighting that everyone is in the same boat, the program fostered collaboration and confidence. This is evident in the following representative comment:

I was thinking also that you're not actually just seen as isolated and in a bubble where you're the only one. What you're actually experiencing is every person is going through exactly the same process and the same insecurities. And that's ... really useful ... Realising you are 
actually part of a big pool, a bigger picture of university ... on lots of levels that was important, not just professionally, but in terms of just being in a group (Sarah).

This togetherness is intimately related to confidence because as one writing tutor has noted, some graduates are 'traumatized by their departments: it’s like they've been beaten up by their departments' (quoted in Levernez, 2001, 57). Part of this ‘traumatisation' emanates specifically from the pressure graduates feel to publish. Emma, for example, spoke about 'being hounded by people in the departments making this point of it's so important and that you publish and they get money ... it's just unwanted pressure. It's not helpful in any way, is it?' In contrast, she said 'hearing the practical stuff [in GRiP was] really, really helpful'. Similarly, John spoke about how early in his candidature his supervisor had said: “ Now you should publish this' and he named the journal ... but I had now idea of how to go about preparing it to that level'. John compared this with his experience in GRiP which gave him 'the confidence that I had a field, a knowledge; that I was competent to work with and just needed to learn the processes'.

Another way in which GRiP diminished graduate isolation was by serving as a counter or supplement to the dyadic student-supervisor relationship. Emma spoke about the difficulties posed by the typically closed nature of this relationship:

I think one of the big things ... is breaking free of that kind of that quite counter-productive relationship with a supervisor that's too private. And I think that if you came and started sharing things with others it would be a good way. Not having a bitch about your supervisor, but actually getting another perspective from a learned community who possibly might know as much about your topic as your supervisor or at least might have heard something about it ... 
So this could also function as ... a positive thing, as a companion to the supervisory relationship.

Emma's comments show that by operating as a place outside of their Schools and departments to share experiences, GRiP gave graduate research candidates a sense of being supported rather than pressured to publish. Providing a non- 'intimidating' (Jane) context in which to practice and conduct the processes that make one a capable and contributing member of an academic community, GRiP played a significant role in building the confidence necessary to venture in to what had until then been perceived as a distant and, in some ways, unwelcoming community. Typically seen as something which arrives with one's first publication, this confidence may in fact be a necessary pre-cursor to, and enabler of, publication for graduates.

\section{Conclusion}

Bearing in mind John Guillory’s (1996) concerns that too vigorous pursuit of professional attainments such as publications may work to ‘deform’ graduate education, we believe that the GRiP program allowed graduate students to achieve the crucial academic professional attainment of publication within a pedagogic structure. The key to the success of the program for the participants lay in the program's enabling and educative orientations: rather than assuming that they 'knew' how to go about the process of writing for publication, the program took them through each step in the process, with guided exercises, peer review and support. For GRiP participants, the program, which focused on their role as writers and supported them in the process of having their work published, appears to have important spin-offs in relation to 
work on their doctoral and masters projects by increasing their writing skills, enhancing their confidence in their disciplinary expertise and in themselves as researchers and writers. Of course, it will be necessary to track these participants through to degree completion, (a process which is under way with the 2005 and 2006 program participants), in order to determine whether this effect is sustained and translates to better completion rates for GRiP participants compared with other research candidates. Whether valuable primarily for taking participants through all the 'baby steps' (Glenn) of writing up research for the very real audience that publication brings, or taking them out of their disciplinary concerns to give them glimpses of the research and publication endeavour as it operates across the disciplines within 'the big picture' (Justine) of the university, a program such as GRiP would appear, on the basis of the evaluations provided by our participants, to serve as a useful adjunct to discipline-based supervision for graduate research students in the humanities and social sciences.

\section{References}

Boud, D. \& Lee, A. (1999) Promoting Research Development through Writing Groups (University of Technology, Sydney); paper presented at the Australian Association for Research in Education, Melbourne, Australia, 1999. Available online at: http://www.aare.edu.au (accessed 3 December 2004)

Damrosch, David (2006) Vectors of Change. In Chris M. Goldie and George E. Walter, eds., Envisoning the Future of Doctoral Education: Preparing Stewards of the Disciplines. Carnegie Essays on the Doctorate. San Francisco, Jossey-Bass, 34-45. 
Galligan, L; Cretchley, P; George, L; McDonald, K; McDonald, J and Rankin, J (2003) Evolution and emerging trends of university writing groups. Queensland Journal of Educational Research, 19(1), 28-41.

Garbus, J. (2005) Tutoring graduate students in the writing center, Academic Exchange Quarterly, 9 (3) 2005, 172-174.

Gere, A. R. (1987) Writing Groups: History, Theory and Implications (Carbondale and Edwardsville: Southern Illinois University Press).

Guillory, J. (1996), Preprofessionalism: What Graduate Students Want, Association of Departments of English Bulletin, 113 (Spring), 4-8.

Heath, T. (2002) A Quantitative Analysis of PhD Students' Views of Supervision, Higher Education Research and Development, 21 (1) 2002, 41-53.

Lee, A. and Boud, D. (2003) Writing Groups, Change and Academic Identity: research development as local practice. Studies in Higher Education, 28( 2), 187-200

Leverenz, C. (2001) Graduate students in the writing center; confronting the cult of non-expertise, in Jane Nelson and Kathy Everz, The Politics of writing centers', (Eds), Portsmouth, New Hampshire, Heninemann/Boynton-Cook, 50-61.

Morss, K. and Murray, R. (2001) Researching Academic Writing within a Structured Program: insights and outcomes. Studies in Higher Education, 26(1), 35-52. 
Page-Adams, D; Cheng, L; Gogineni, A; Shen, C (1995) Establishing a Group to Encourage Writing for Publication among Doctoral Students. Journal of Social Work Education, 31(3), 402-407.

Sheely, S. (1996) A degree by any other name: An historical reflection on traditional and non-traditional PhD programs, Different Approaches: Theory and Practice in Higher Education. Proceedings HERDSA Conference 1996. Perth, Western Australia, 8-12 July. http://www.herdsa.org.au/confs/1996/sheely2.html 